\title{
Reduced Order Dynamic Model of Permanent Magnet and HTSC Interaction in an Axisymmetric Frame
}

\author{
Clay S. Hearn, Siddharth B. Pratap, Senior Member, IEEE, Dongmei Chen, Member, IEEE, ASME \\ and Raul G. Longoria, Member, IEEE, ASME.
}

\begin{abstract}
This paper presents a reduced order model for a permanent magnet and high temperature superconductor (HTSC) in an axisymmetric frame. This model is formulated as a bondgraph to be used for system models such as lift bearing applications, where the nonlinear force-displacement interactions are important for stability analysis and control design. The reduced order model is based on the mechanical and electromagnetic interaction between a permanent magnet and bulk HTSC. Performance of the proposed reduced order model is compared to FEM analysis and experimental tests to confirm the static and transient performance.
\end{abstract}

Index Terms-high temperature superconductor, bond graph, HTSC bearings

\section{INTRODUCTION}

$\mathrm{H}$ IGH temperature superconducting (HTSC) bearings are actively being researched and implemented in levitation applications where minimal losses are required, such as flywheel energy storage. Boeing has developed a HTSC thrust bearing to suspend a $132 \mathrm{~kg}$ rotor for a $5 \mathrm{kWh}$ flywheel [1], and the Adelwitz Technologiezentrum has also developed bearings for $600 \mathrm{~kg}$ rotors [2]. In addition to achieving sufficient load carrying capacity, implementation of passive PM-HTSC bearing systems must deal with low damping characteristics which may limit rotor speeds [3].

The principal design for a HTSC bearing utilizes the stable levitation of a permanent magnet (PM) above a bulk superconductor. Generally, type II superconductors are used for these applications. These superconductors exhibit a lower and upper critical field below a critical temperature. At low magnetic fields, these superconductors exhibit perfect diamagnetism and reject all flux [4]. Above this lower critical field, type II superconductors enter a mixed state where flux lines can penetrate the material and induce circulating currents at pinning centers, which flow at a critical current density

Manuscript received August 27, 2012. This work was supported by Stanford University through the Global Climate and Energy Project (GCEP).

C. S. Hearn and S. B. Pratap are with the Center for Electromechanics at the University of Texas at Austin, Austin, TX 78758 USA (e-mail: hearn@cem.utexas.edu; s.pratap@cem.utexas.edu).

D. Chen and R.G. Longoria are with the Dept. of Mechanical Engineering at the University of Texas at Austin, Austin, TX 78712 USA. (e-mail: dmchen@me.utexas.edu; r.longoria@mail.utexas.edu). without resistance [5], [6]. Above the upper critical field, superconductivity is lost, and the material becomes resistive.

Currently there is a lack of resources in the literature for developing physics-based, reduced order models which can be used by engineers to design and control HTSC bearings. These models should be able to predict the local bearing stiffness and dynamic response. Bean first proposed the critical state model in which current flow in a bulk HTSC is either zero or equal to the magnitude of the critical current density [7]. The critical state model has been used and verified in a finite element method (FEM) which utilizes Maxwell's field equations to determine field distribution and resulting forces [8]. Although FEM is the best way to model the interaction between a permanent magnet and a bulk HTSC, these models are computationally intensive and may not be appropriate for initial stages of a design process where many iterations may be required. These models are also not applicable for describing transient behavior with respect to system level control design. There is a need to develop a low order HTSC model to describe transient behavior with much less computation expense.

Tests performed by Moon and Hikihara demonstrated a force-displacement hysteresis curve and nonlinear drift response to vibration in the interaction between a permanent magnet and cooled HTSC [9], [10]. These authors also presented a non-dimensional, nonlinear dynamic model based on friction dynamics [11] rather than on the electrodynamics of the system to describe the PM-HTSC interaction.

Mirror image and advanced mirror image models have been developed by Kordyuk [12] and by Hull and Cansiz [13], [14]. This methodology modeled the permanent magnet as a dipole with a stationary image on the other side of the bulk HTSC surface, and a diamagnetic image that mirrored the position of the permanent magnet. This technique can be used to characterize the stiffness and force relation between a permanent magnet and bulk HTSC, but it does not consider the energy loss mechanisms that contribute to the dynamics and hysteretic behavior of the force-displacement curve [15].

Another method presented by Davey et al [16] modeled the bulk HTSC as a series of nested superconducting elements or rings. The superconducting elements are modeled in accordance to the Critical State model, in which the current flowing though the rings is equal either to zero or to the 
magnitude of the critical current density. Davey used this technique to perform quasi-static solutions which predict trapped fields due to flux pumping.

This paper will develop a dynamic model of the forcedisplacement interaction between a permanent magnet and a bulk HTSC to describe the system transient behavior. This method will be an extension of the work proposed by Davey in which discrete rings are used to model the PM and bulk HTSC. This model considers vertical motion in an axisymmetric frame, and includes the energy storage and loss mechanisms inherent to the system. A bond graph formulation is presented to establish the modeling framework for the bearing system. The mathematic formulation of each bond will lead to the system dynamic equations. This modulated model can be added to a larger system model framework. Dynamic drop testing was then performed to verify model prediction.

\section{Model DeVELOPMENT}

\section{A. Description}

The HTSC puck is modeled as discrete, nested, superconducting rings shorted on themselves, Fig. 1. Discrete current loops on the surface of the permanent magnet are used to represent the equivalent surface currents and resulting magnetic fields in free space. This model assumes the permanent magnet puck is concentric to the bulk HTSC, which allows the use of an axisymmetric model.

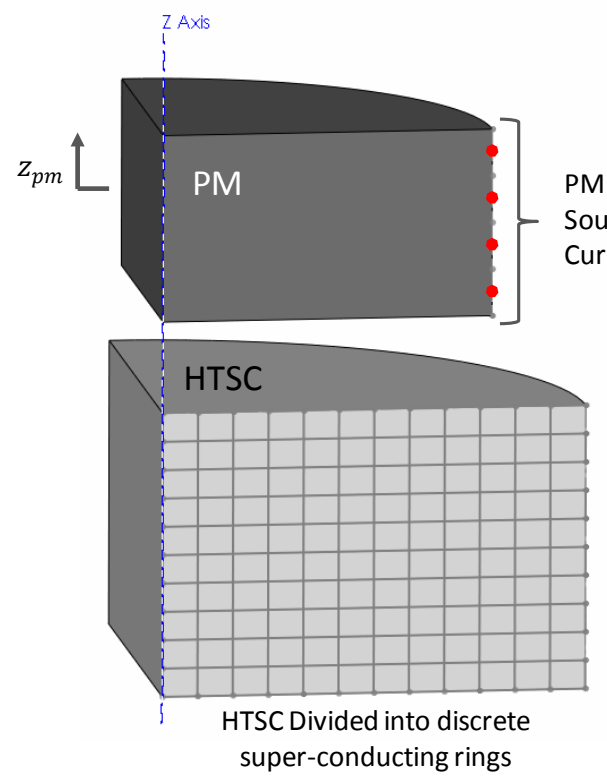

Fig. 1. Schematic layout of permanent magnet and a high temperature superconductor

A bond graph is used here to represent the interaction between the permanent magnet and the bulk HTSC. Bond graphs are highly useful for modeling systems across multiple energy domains, such as the mechanical and magnetic coupling of the PM-HTSC system. A proposed bond graph for the system under study is shown in Fig. 2. From the far left 1junction of the proposed bond graph, the equation of motion of the levitated mass in the z-direction can be derived by (1), where $F_{r}$ represents any mechanical frictional losses that may act on the mass, and $F_{p m}$ represents the reactive forces generated by the magnetic interaction between the PM and bulk HTSC. As will be shown, these reactive forces are a function of the PM velocity, $v_{p m}$, and vertical position over the mass, $z_{p m}$. The right-hand portion of the bond graph can be thought of as a nonlinear mechanical spring, which is represented by a capacitive (C) element in bond graph terminology. Within this capacitive element are a series of gyrator elements (G) and interconnected 1-junctions which represent the flux linkages of the superconducting rings used to model the bulk HTSC.

$$
M \frac{d v_{p m}}{d t}=-M g-F_{r}\left(v_{p m}\right)-F_{p m}\left(v_{p m}, z_{p m}\right)
$$

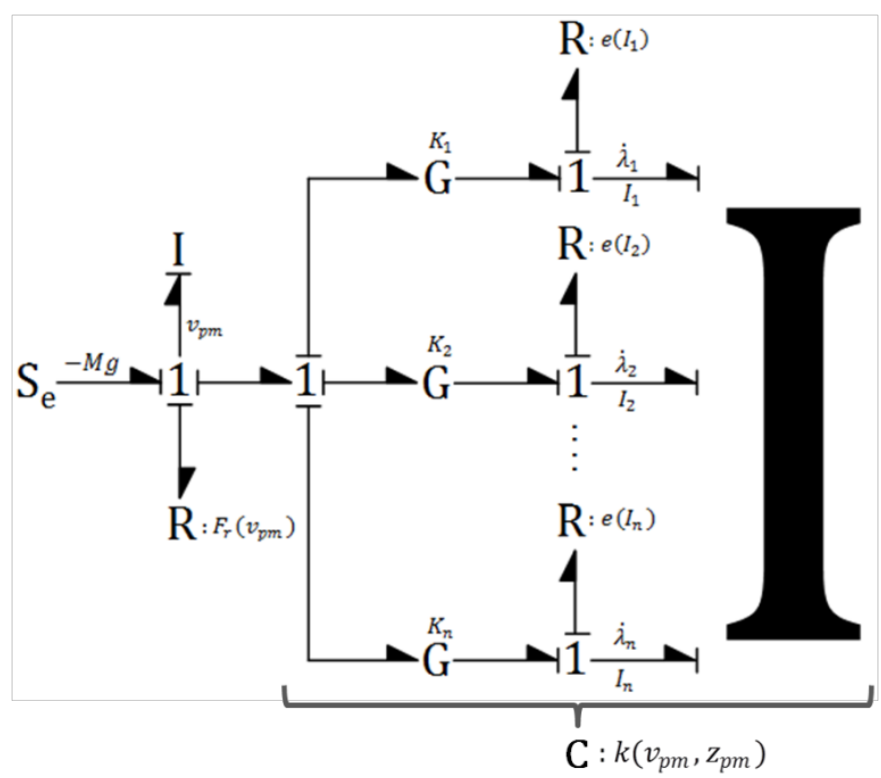

Fig. 2. Proposed bond graph for modeling vertical motion of a permanent magnet over a bulk HSTC

\section{B. Inductance Modeling}

The superconducting ring is modeled as $\mathrm{n}$ discrete superconducting sub-rings with current $I_{i}$ and flux linkage $\lambda_{i}$. The order of the model will equal the number of sub-rings used, plus the other mechanical states. The total flux linkage for each sub-ring is a summation of the self induced flux with the mutually linked fluxes from the neighboring sub-rings, and the external flux linkage from the permanent magnet, (2). The inductance matrix, $\boldsymbol{L}$, is symmetric with self inductances on the diagonal, and mutual inductance terms on the off-diagonals. The external flux linkage from the permanent magnet, $\phi_{i}\left(z_{p m}, z_{i}\right)$, is a function of the axial displacement between the permanent magnet, $z_{p m}$, and the superconducting ring, $z_{i}$. As shown by Fig. 2, this relationship between the flux linkages, circulating currents, and mutual inductances is represented by the multi-port $I$ element [17]. 


$$
\left[\begin{array}{c}
\lambda_{1} \\
\lambda_{2} \\
\vdots \\
\lambda_{n}
\end{array}\right]=\left[\begin{array}{cccc}
L_{11} & L_{12} & \cdots & L_{1 n} \\
L_{12} & L_{22} & \cdots & L_{2 n} \\
\vdots & \vdots & \ddots & \vdots \\
L_{1 n} & L_{2 n} & \cdots & L_{n n}
\end{array}\right]\left[\begin{array}{c}
I_{1} \\
I_{2} \\
\vdots \\
I_{n}
\end{array}\right]+\left[\begin{array}{c}
\phi_{1}\left(z_{p m}, z_{1}\right) \\
\phi_{2}\left(z_{p m}, z_{2}\right) \\
\vdots \\
\phi_{n}\left(z_{p m}, z_{n}\right)
\end{array}\right]
$$

\section{Resistance Modeling}

In addition to the mutual flux linkages represented by the multi-port I-element, each ring also includes a nonlinear resistive element to model the rapid rise in resistivity once the critical current density is exceeded. Instead of implementing the critical state model, a power law, described by Equation (3), can be used here to characterize the nonlinear relationship between ring current and voltage loss, $e_{i}$, [18], [19]. Equation (3) has been modified from Grilli to represent the current system. In (3), $r_{i}$ is the mean radius of the conducting ring, i, $A_{i}$ is the cross sectional area of the conducting ring, $E_{c}$ is the threshold electric field, and $J_{c}$ is the critical current density. The threshold electric field is set at $1 \mu \mathrm{V} / \mathrm{cm}$ per Grilli. The exponential factor $n$ determines the rate of rise in resistivity once the critical current density is exceeded. Grilli suggests values of $n$ to range from 5 to 30 .

$$
e_{i}\left(I_{i}\right)=2 \pi r_{i} E_{c}\left(\frac{\left|I_{i}\right|}{A_{i} J_{c}}\right)^{n} \operatorname{sign}\left(I_{i}\right)
$$

\section{Gyrator Modeling}

Gyrator elements in Fig. 2 are used to represent the Faraday induction (4) on the superconducting rings. The induced electromotive force, $\varepsilon_{i}$, on each ring is equal to the time varying magnetic flux, $\phi_{i}$, through the ring due to axial movement of the PM. The following procedure will outline a methodology to determine the time varying flux in terms of the PM position and velocity.

$$
\varepsilon_{i}=-\frac{d \phi_{i}}{d t}
$$

As shown by Fig. 1, the magnetic fields produced by the PM are represented by discrete current loops located on the surface. The goal of this method is to replicate the magnetic field generated by the axial position of the permanent magnet. This method does not intend to model the electrodynamics of the permanent magnet itself, since the exterior currents are assumed as a fixed source. Fig. 3 shows a schematic representation, where there is a fixed source current, $I_{s}$, at axial position $z_{s}$ with radius $a_{s}$, which interacts with a passive conducting ring at axial position $z_{i}$ and radius $r_{i}$.

The magnetic flux that links the conducting ring, $i$, at axial position $z_{i}$ and radius $r_{i}$ due to the source current at $z_{s}$ can be calculated by taking the line integral of the magnetic potential at the position of the conducting ring, per Stoke's Theorem (5). Since the problem is axisymmetric, the magnetic potential, $A_{i}$, is constant around the circumference of the conducting ring.

$$
\phi_{s i}=\oint A_{s i} d l_{i}=2 \pi r_{i} A_{s i}
$$

The magnetic potential due to circular current loop, as shown in Fig. 3, can be calculated by the techniques derived by Smythe [20]. The magnetic potential due to current loop $I_{s}$, with radius $a_{s}$, at axial location $z_{s}$, can be determined at radius $r_{i}$ and axial location $z_{i}$ by (6).

$$
A_{s i}=\frac{\mu_{0} I_{s}}{\pi k_{s i}}\left(\frac{a_{s}}{r_{i}}\right)^{\frac{1}{2}}\left(\left(1-\frac{1}{2} k_{s i}^{2}\right) K\left(k_{s i}\right)-E\left(k_{s i}\right)\right)
$$

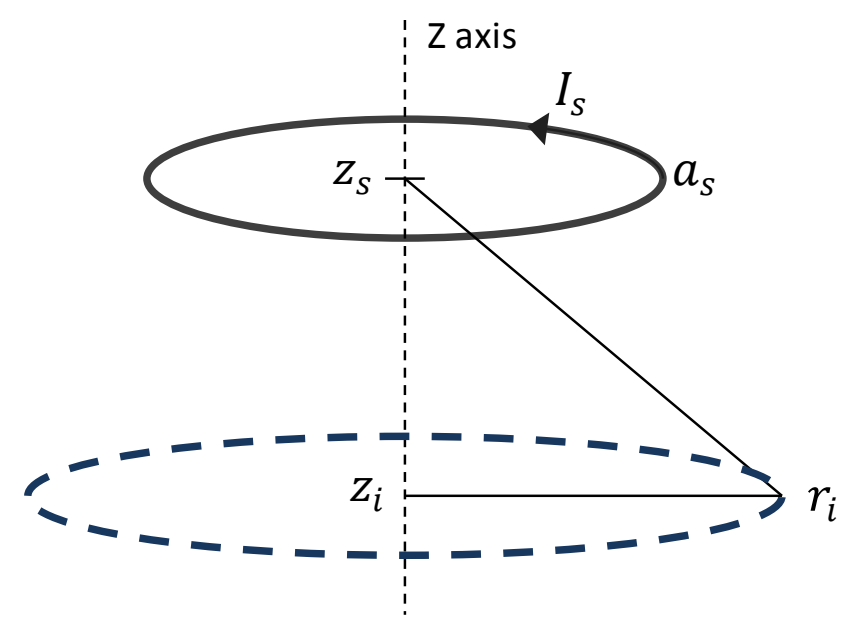

Fig. 3. Schematic of a fixed filament current loop at axial position $z_{s}$ with constant current $I_{s}$, to a passive conducting ring at axial position $z_{i}$ and radius $r_{i}$

The functions $K\left(k_{s i}\right)$ and $E\left(k_{s i}\right)$ are the complete elliptical integrals of the first and second kind with modulus $k_{s i}$. The modulus is calculated by (7).

$$
k_{s i}=\sqrt{\frac{4 a_{s} r_{i}}{\left(a_{s}+r_{i}\right)^{2}+\left(z_{i}-z_{s}\right)^{2}}}
$$

Using (5) through (7), the magnetic flux from the PM linking each ring can be calculated. If multiple source currents are used to model the permanent magnet, superposition can be used to sum the magnet fluxes linking each ring. Now that the expressions for the magnetic flux have been stated, the next step is to determine the time varying magnetic flux as a function of the permanent magnet velocity, $v_{p m}$. The time varying magnetic flux linking a conducting ring can be evaluated by (8), where the axial gradient of the flux is multiplied by the axial velocity of the permanent magnet. Per Faraday's law, the negative of the spatial gradient in the $\mathrm{z}$ direction of magnetic flux is represented by the gyrator factor $K_{i}$ (9), as shown in the bond graph in Fig. 2.

$$
\frac{d \phi_{s i}}{d t}=\frac{d \phi_{s i}}{d z_{s}} \frac{d z_{s}}{d t}=\frac{d \phi_{s i}}{d z_{s}} v_{p m}(t)
$$




$$
K_{i}=-\frac{d \phi_{s i}}{d z_{s}}
$$

The axial gradient of magnetic flux is determined by further derivation. First, take the derivative of (5) with respect to $z_{s}$, which yields (10).

$$
\frac{d \phi_{s i}}{d z_{s}}=2 \pi r_{i} \frac{d A_{s i}}{d z_{s}}
$$

The gradient of magnetic potential with respect to axial position is calculated by (11).

$$
\begin{aligned}
\frac{d A_{s i}}{d z_{s}}=\frac{\mu_{0} I_{s}}{\pi k_{s i}}\left(\frac{a_{s}}{r_{i}}\right)^{\frac{1}{2}} & {\left[( 1 - \frac { 1 } { 2 } k _ { s i } ^ { 2 } ) \left(\frac{d K\left(k_{s i}\right)}{d z_{s}}\right.\right.} \\
& \left.-\frac{K\left(k_{s i}\right)}{k_{s i}} \frac{d k_{s i}}{d z_{s}}\right) \\
& +\left(\frac{E\left(k_{s i}\right)}{k_{s i}}-k_{s i} K\left(k_{s i}\right)\right) \frac{d k_{s i}}{d z_{s}} \\
& \left.-\frac{d E\left(k_{s i}\right)}{d z_{s}}\right]
\end{aligned}
$$

Equation (12) shows the derivative of the elliptical modulus with respect to $z$.

$$
\frac{d k_{s i}}{d z_{s}}=-\frac{k_{s j}^{3}\left(z_{s}-z_{i}\right)}{\left(4 a_{s} r_{i}\right)}
$$

The derivatives of the elliptical integrals can be found in Smythe [20] and calculated by the formulations in (13) and (14).

$$
\begin{gathered}
\frac{d K\left(k_{s i}\left(z_{s}\right)\right)}{d z_{s}}=\frac{d K}{d k_{s i}} \frac{d k}{d z_{s}} \\
\frac{d E\left(k_{s i}\left(z_{s}\right)\right)}{d z_{s}}=\frac{d E}{d k_{s i}} \frac{d k}{d z_{s}} \\
\frac{d K}{d k_{s i}}=\frac{E}{k_{s i}\left(1-k_{s i}^{2}\right)}-\frac{K}{k_{s i}} \\
\frac{d E}{d k_{s i}}=\frac{E}{k_{s i}}-\frac{K}{k_{s i}}
\end{gathered}
$$

\section{E. One Junction Modeling of Total Force}

Based on the bond graph representation of Fig. 2, the total force on the permanent magnet can be calculated by reverse summation through the gyrator elements utilizing the gyrator factors, $K_{i}(15)$.

$$
F_{p m}=\sum_{i=1}^{n} K_{i} I_{i}=\sum_{i=1}^{n}-\frac{d \phi_{s i}}{d z_{s}} I_{i}
$$

To verify this relationship, the force on the permanent magnet can also be calculated based on the co-energy of the magnetic field. This force can be calculated by taking the derivative of the magnetic co-energy, $W_{m}^{\prime}$, with respect to axial position $z_{s i}$ as shown by (16), with respect to the permanent magnet, for equal and opposite forces [21].

$$
F_{p m}=-\frac{d W_{m}^{\prime}}{d z_{s}}
$$

The magnetic co-energy can be calculated by taking the summation integral of the flux linkage given in (1) with respect to all the conducting ring currents (17).

$$
W_{m}^{\prime}=\sum_{i=1}^{n} \int_{0}^{I_{i}}\left(\sum_{j=1}^{n} L_{i j} I_{j}\right)+\phi_{i} d I_{i}
$$

From evaluating the integrals in (17), it is clear that the external flux linkage, $\phi_{i}$, is the only variable that is a function of the axial position. Therefore, taking the derivative of the coenergy with respect to axial position yields the expression in (18) which is the same as derived from the bond graph formulation.

$$
F_{p m}=-\frac{d W_{m}^{\prime}}{d z_{s}}=\sum_{i=1}^{n}-\frac{d \phi_{s i}}{d z_{s}} I_{i}
$$

\section{F. Modeling Summary}

The complete set of dynamic equations for the levitating PM with mass $M$ is given by (19). The model order is dependent on the number of rings used to model the bulk HTSC, thus giving $n$, current states in addition to the velocity and position states of the PM mass.

$$
\left.\begin{array}{c}
\frac{d z_{p m}}{d t}=v_{p m} \\
\frac{d v_{p m}}{d t}=-g-\frac{F_{r}\left(v_{p m}\right)}{M}+\frac{1}{M} \sum_{i=1}^{n} \frac{d \phi_{s i}}{d z_{s i}} I_{i} \\
{\left[\begin{array}{c}
\frac{d I_{1}}{d t} \\
\frac{d I_{2}}{d t} \\
\vdots \\
\frac{d I_{n}}{d t}
\end{array}\right]=-L^{-1}\left[\begin{array}{c}
e_{1}\left(I_{1}\right) \\
e_{2}\left(I_{2}\right) \\
\vdots \\
e_{n}\left(I_{n}\right)
\end{array}\right]-\left[\begin{array}{c}
\frac{d \phi_{s 1}}{d z_{s}} \\
\frac{d \phi_{s 2}}{d z_{s}} \\
\vdots \\
\frac{d \phi_{s n}}{d z_{s}}
\end{array}\right]}
\end{array}\right]
$$

The inductance matrix, $\boldsymbol{L}$, is calculated using a thin filament assumption for each ring in the bulk HTSC. The elements of the inductance matrix from (2) are calculated by (20) using methods outlined by Smythe [20] to determine self and mutual inductances. In (20), $w_{i}$ is the width of a filament ring conductor in the bulk HTSC.

$$
L_{i j}=\left\{\begin{array}{cc}
i=j & \mu_{0} r_{i}\left(\ln \left(\frac{16 r_{i}}{w_{i}}-2\right)+0.25\right) \\
i \neq j & \frac{\mu_{0}}{\pi k_{i j}}\left(\frac{r_{i}}{r_{j}}\right)^{\frac{1}{2}}\left(\left(1-\frac{1}{2} k_{i j}^{2}\right) K\left(k_{i j}\right)-E\left(k_{i j}\right)\right)
\end{array}\right.
$$




\section{FEM COMPARISON}

To initially verify performance of the reduced order model, comparisons were made to the finite element method (FEM). The FEM algorithm, as described by Alsono and Coombs [8] which utilizes the critical state model, was used for this analysis.

The system used in this study consists of a solid PM plunging through an HTSC ring, Fig. 4. For this simulation, the $\mathrm{PM}$ is given a known velocity profile and the goal is to

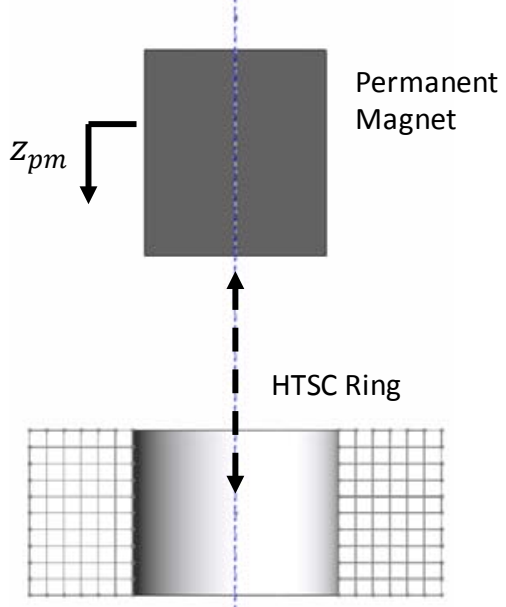

Fig. 4. Schematic layout of PM and HTSC to verify reduced order model against FEA analysis

verify the reaction force between the two methods. The PM has a radius of $4.41 \mathrm{~mm}$ and a height of $10 \mathrm{~mm}$. The magnet was modeled as 10 equally spaced current loops of 9,080 A, relating to a magnetic coercive strength of $908 \mathrm{kA} / \mathrm{m}$. The HTSC ring has an inner radius of $5 \mathrm{~mm}$, and outer radius of 10 $\mathrm{mm}$, with a height of $8 \mathrm{~mm}$. The HTSC ring is 48 equally spaced conducting rings, which translates into a $48^{\text {th }}$ order model. For this simulation, the HTSC ring is assumed to be a chemically pinned material, with a critical current density of $6.666 \mathrm{e} 7 \mathrm{~A} / \mathrm{m}^{2}$. A exponential value of $n=16$ was used for the non-linear voltage loss.

For the simulation, the permanent magnet moves through the HTSC ring at a low constant velocity of $0.467 \mathrm{~mm} / \mathrm{s}$ between axial positions of $+30 \mathrm{~mm}$ to $-40 \mathrm{~mm}$, where the HTSC ring is centered at $0 \mathrm{~mm}$. Three full cycles of movement through the HTSC ring was performed for this analysis. Fig. 5 shows a comparison of force versus axial displacement results for the reduced order dynamic model to a high order FEM method.

The reduced order model shows good matching of the forcedisplacement profile calculated by the FEM analysis. The initial descent of the magnet through the HTSC ring results in the highest repulsive force, which turns into an attractive force once the magnet passes through the ring. Fig. 6 plots the current density of each conducting ring with respect to axial position. Due to rapidly increasing resistivity, currents saturate near the set critical current density, which results in trapped magnetic fields by the HTSC ring.

\section{EXPERIMENTAL VALIDATION}

To verify dynamic performance of the model, a test setup was constructed to evaluate the response of the permanent magnet falling over a bulk HTSC. The purpose of this drop test is to illicit a dynamic response from the system by means of a step input, where the weight of the PM, and any additional mass, is quickly transferred to the magnetic interaction between the PM and HTSC as described by the proposed model.

A picture of the test setup is shown in Fig. 7. A high strength N45 neodymium magnet, with $38.1 \mathrm{~mm}$ OD x $6.4 \mathrm{~mm}$ ID x $12.7 \mathrm{~mm} \mathrm{H}$, is initially positioned over bulk HTSC by a spacer bar. The bulk HTSC is YBa2Cu3O7 and measures 47.5

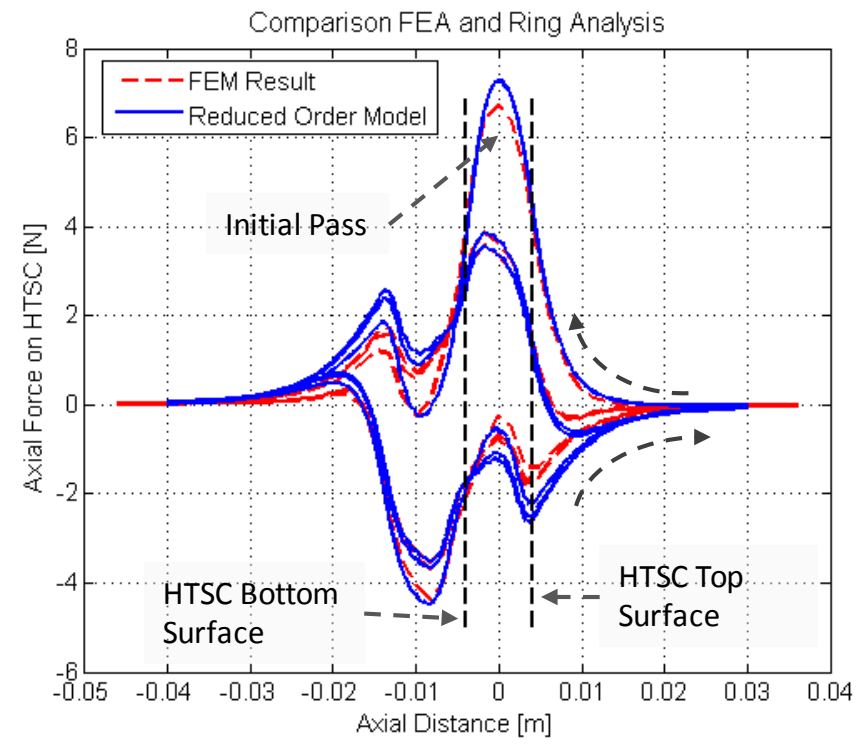

Fig. 5. Comparison of force displacement profile between reduced order dynamic simulation versus high order FEM analysis

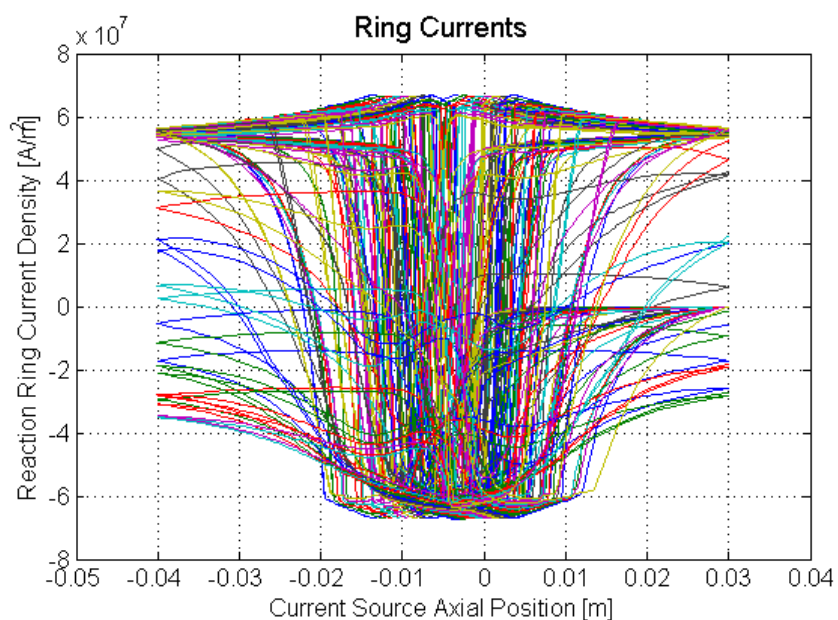

Fig. 6. Ring current density versus axial position of permanent magnet

mm OD x 15 mm H. A composite-glass G-10 rod runs through the center of the PM to ensure that the motion of the PM is in the vertical direction over the bulk HTSC, and remains centered. A G-10 plate is also attached to the top of the PM to provide a measurement surface for the IDEC MX1A-B12 laser displacement sensor. The permanent magnet with G-10 plate had an initial weight of $117.8 \mathrm{~g}$. Two additional blocks of beryllium copper were added to the top of the magnet to bring the total mass to $520 \mathrm{~g}$. Although beryllium copper has a 
conductivity of $20-28 \%$ IACS [22], the blocks are travelling with the permanent magnet and should not experience any changing magnetic fields that would induce significant currents.

Spacer rods of varying height, $15 \mathrm{~mm}, 20 \mathrm{~mm}$, and $25 \mathrm{~mm}$, were used to set the initial height of the permanent magnet over the bulk HTSC at room temperature. Once the position was set, liquid nitrogen was added to the basin holding the bulk HTSC to bring the temperature down to $77 \mathrm{~K}$. After the HTSC was successfully field cooled, the spacer bar initially supporting the weight of the permanent magnet was removed

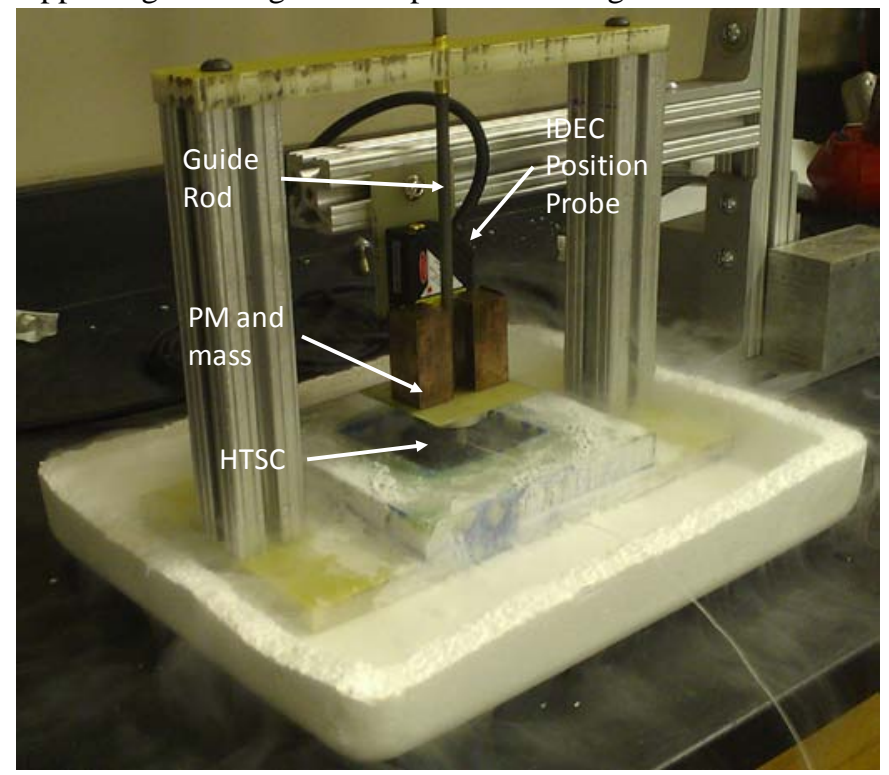

Fig. 7. Dynamic drop test setup

to allow it to drop over the bulk HTSC. The IDEC probe measured position which was recorded at $1000 \mathrm{~Hz}$ rate.

\section{RESULTS AND ANALYSIS}

Comparisons of model predictions and recorded test data were made by using the model to simulate the same conditions of initial set height and loaded mass as the tests. Due to the friction between the permanent magnet and G-10 guide rod, the system had a damped response to the weight transfer. This mechanical friction was added to the model as a combination of coulomb and viscous friction with fixed constant parameters, $F_{c}$ and $B_{v}$ (19). These parameters were roughly adjusted to match the time domain dynamic response.

$$
F_{r}\left(v_{p m}\right)=F_{c} \cdot \operatorname{sign}\left(v_{p m}\right)+B_{v} v_{p m}
$$

The bulk HTSC was modeled as 130 discrete superconducting rings. A critical current density for the chemically pinned puck of $9.5 \mathrm{kA} / \mathrm{cm}^{2}$ was assumed, which was at the low end of the manufacturer's specifications. The permanent magnet was modeled with 11 discrete current elements, each on the ID and OD surfaces, which correspond to $1030 \mathrm{kA} / \mathrm{m}$. The measured peak fields in air of the permanent magnet at $0.5 \mathrm{~mm}$ above the surface was $0.4 \mathrm{~T}$, as shown in Fig. 8. These field measurements correspond well to an ANSYS Maxwell FEM model of N45 magnetic material, and a model of the magnet modeled by surface currents, as shown in Fig. 9.

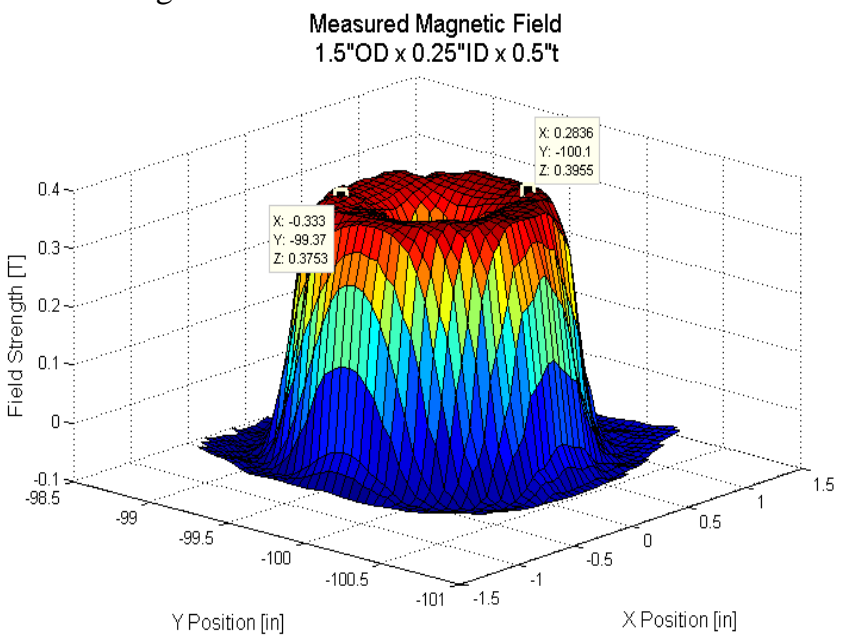

Fig. 8. Measured magnetic field from $0.5 \mathrm{~mm}$ above permanent magnet

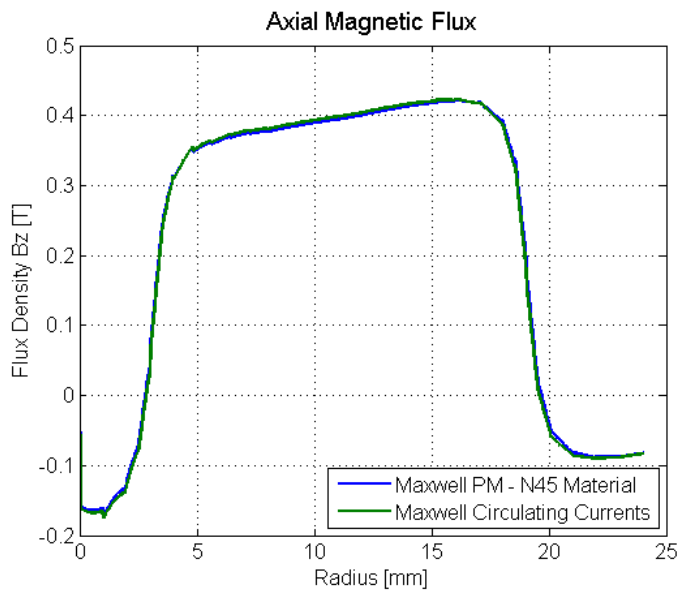

Fig. 9. Comparisons from ANSYS Maxwell FEM model of N45 PM material versus representation of the PM utilizing surface currents.

Figures 10, 11, and 12 show comparisons between model prediction and experimental test for drops at $15 \mathrm{~mm}, 20 \mathrm{~mm}$, and $25 \mathrm{~mm}$ heights respectively. The time-domain response, shown in the left pane of these figures, shows the model tracks well to experimental results. The largest errors come from inaccurate modeling of the friction between the magnet and guide rod, which was only roughly estimated. A fast Fourier transform was also performed to verify oscillation frequency, which is shown in the right side of Figs. 10, 11, and 12. The FFT shows that the model presented matches the frequency response and local stiffness of the system relatively well.

This model technique is applicable to any configuration of permanent magnets and HTSC in an axisymmetric frame. The effective axial spring rate and levitation forces can be characterized with this model to determine bearing lift forces for a thrust and journal bearing configurations. Although this modeling technique is currently limited to vertical motion, this technique is applicable to analysis of permanent magnets and bulk HTSC for thrust bearing design. Due to tight clearances between rotor and stator components, large excursions in the radial direction would not be expected. 

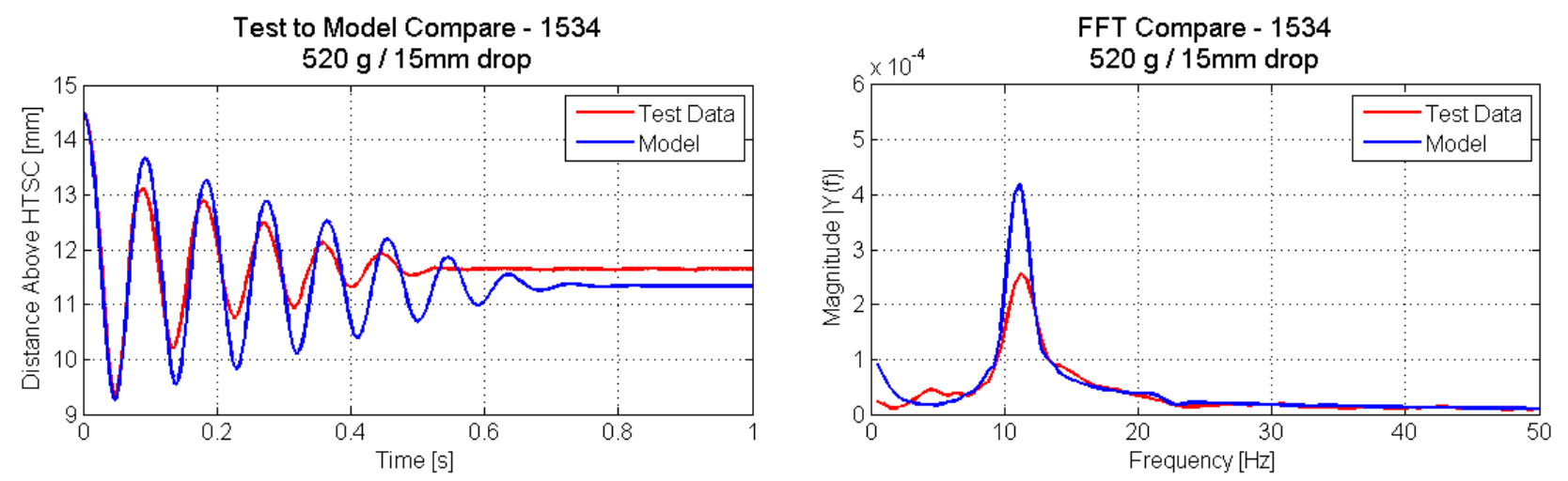

Fig. 10. Model and test comparison at set height of 15mm above HTSC. Model and test both show frequency response of $11.23 \mathrm{~Hz}$
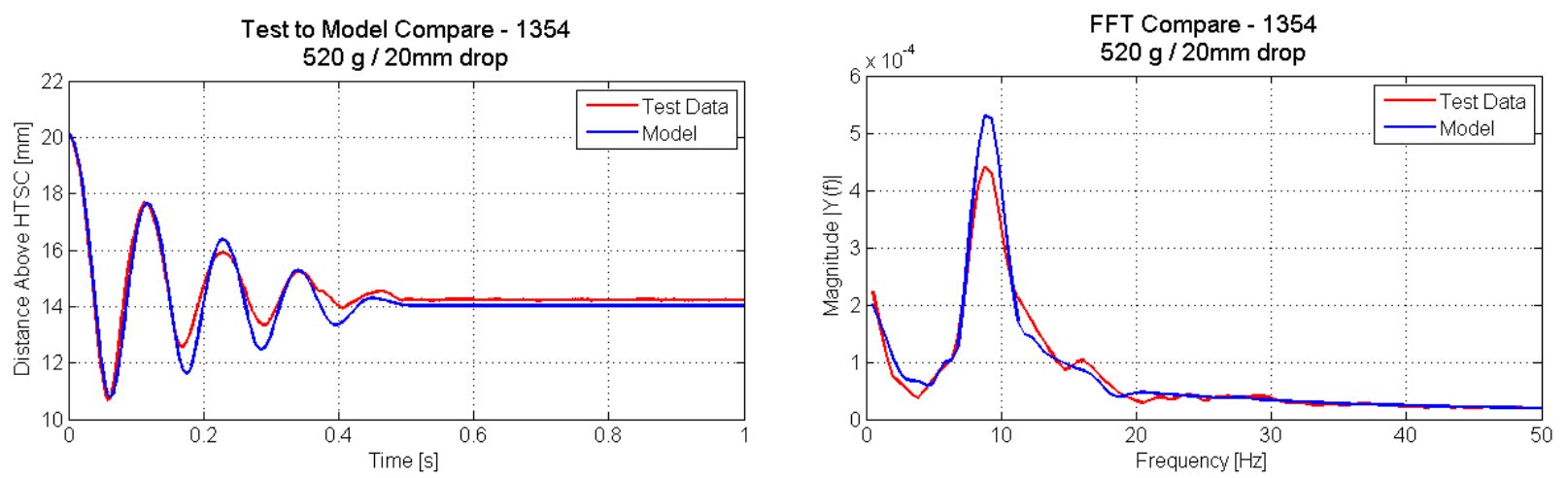

Fig. 11. Model and test comparison at set height of 20mm above HTSC. Model and test both show frequency response of $8.79 \mathrm{~Hz}$
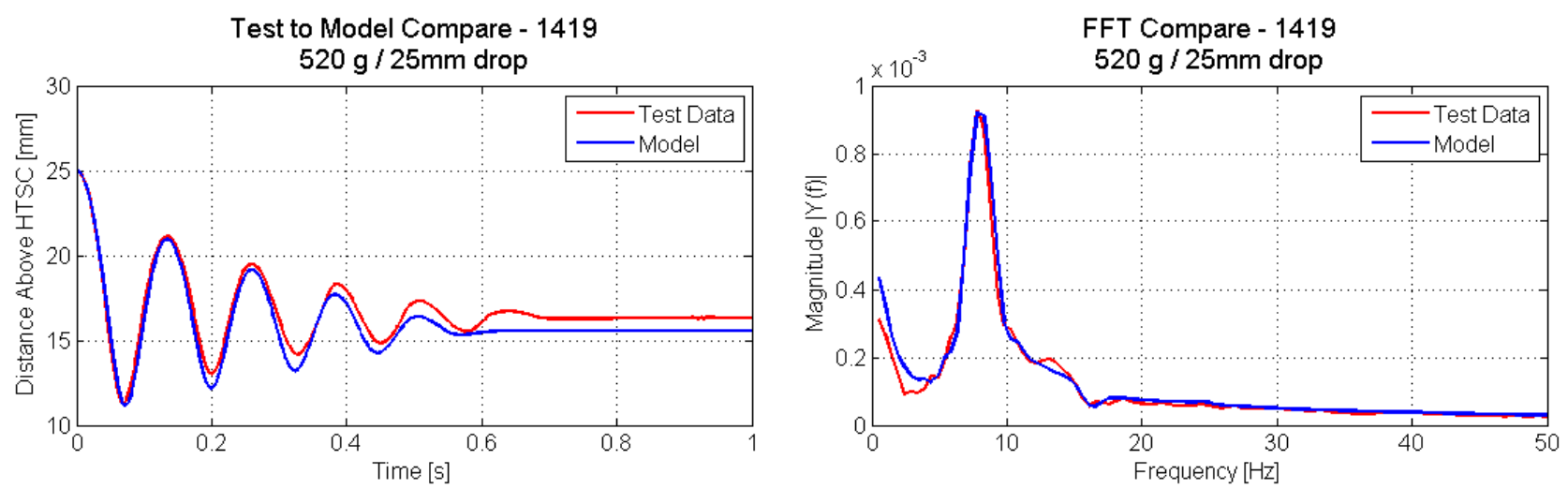

Fig. 12. Model and test comparison at set height of 25mm above HTSC. Model and test both show frequency response of $7.81 \mathrm{~Hz}$

\section{CONCLUSION}

This paper presents the methodology for modeling the dynamic interaction between a permanent magnet and HTSC in an axisymmetric frame and the subsystem model is formulated using a bond graph. This approach promotes the use of the model as part of a more complex bearing system, and the methodology significantly reduces computational time and model-order over FEM techniques. The model captures the stiffness and dynamic behaviors that are important for system control design.

The proposed reduced order model was tested against FEM analysis and experimental data. This model showed good matching of the force-displacement profile to FEM predications for a novel combination of PM and HTSC ring. In addition, model predictions are given to compare performance to results from an experimental drop test designed to elicit system dynamic response. The results show that the proposed model is in agreement with experimental test results of the dynamic interaction between a permanent magnet and bulk HTSC.

This model allows designers to have the first ever capability to quickly calculate vertical lifting capacity and dynamic response for a potential thrust bearing design. The model also enables a control design for the bearing system since it accurately predicts the transient behavior of a PM-HTSC bearing system with a low-order system representation. 


\section{REFERENCES}

[1] M. Strasik, J. R. Hull, J. A. Mittleider, J. F. Gonder, P. E. Johnson, K. E. McCrary and C. R. McIver, "An Overview of Boeing Flywheel Energy Storage Systems with High Temperature Superconducting Bearings," Superconducting Science and Technology, vol. 23, 2010.

[2] F. N. Werfel, U. Floegel-Delor, R. Rothfeld, T. Riedel, B. Goebel, D. Wippich and P. Schirrmeister, "Superconductor Bearings, Flywheels and Transportation," Superconductor Science and Technology, vol. 25, 2012.

[3] T. M. Mulcahy, J. R. Hull, K. L. Uherka, R. G. Abboud and J. J. Juna, "Test Results of a 2 kWh Flywheel Using Passive PM and HTS Bearings," IEEE Transactions on Applied Superconductivity, vol. 11, no. 1, pp. 1729-1732, March 2001.

[4] Francis C. Moon. Superconducting Levitation: Applications to Bearings and Magnetic Transportation. pp. 79, John Wiley \& Sons, New York, 1994.

[5] K.B. Ma, Y.V. Postrekhin, and W.K. Chu. Superconducting and Magnetic Levitation Devices. Review of Scientific Instruments, Vol. 74, No. 12, Dec. 2003

[6] G. Ries and F. Werfel. Supraleitende Magnetlager In der Schwebe. Phys. Unserer Zeit. Vol 35, No 3, 2004.

[7] C.P. Bean. Magnetization of High Field Superconductors. Rev. Mod. Phys. 36, pp. 31-39, 1964.

[8] D.R. Alonso, T.A. Coombs, and A.M. Campbell. "Numerical Analysis of High-Temperature Superconductors with the Critical State Model”. IEEE Transactions on Applied Superconductivity, vol. 14, no. 4, pp. 20532063, Dec. 2004.

[9] T. Hikihara and F. C. Moon, "Levitation drift of a magnet supported by a high-Tc superconductor under vibration," Physica , vol. 250, pp. 121127, 1995.

[10] T. Hikihara and F.C. Moon Chaotic Levitated Motion of a Magnet Supported by Superconductor. Physics Letters A. 191 pp. 279-284, 15 Aug. 1994.

[11] A. Ruina. Slip Instability and State Variable Friction Laws. Journal of Geophysical Research, Vol. 88, No. B12 pp. 359-370. Dec. 10, 1983.

[12] A. A. Kordyuk, "Magnetic Levitation for Hard Superconductors," Journal of Applied Physics, vol. 83, pp. 610-612, 1998.

[13] J. R. Hull and A. Cansiz, "Vertical and Lateral Forces Between a Permanent Magnet and a High Temperature Superconductor," Journal of Applied Physics, vol. 86, no. 11, pp. 6396-6404, 1999.

[14] A. Cansiz, J. R. Hull and Ö. Gundogdu, "Translational and Rotational Dynamic Analysis of a Superconducting Levitation System," Superconducting Science and Technology, vol. 18, pp. 990-996, 2005.

[15] D. Karnoop. Computer Models of Hysteresis in Mechanical and Magnetic Components. Journal of the Franklin Institute, Vol. 316, Is. 5, pp. 405-415, Nov. 1983.

[16] K.R. Davey, R. Weinstein, and R. Sawh. Activation of Trapped Field Magnets by Flux Pumping. IEEE Conference on Electromagnetic Field Computation, 2010.

[17] Karnopp, Dean C., Margolis, Donald L., and Rosenberg, Ronald C. System Dynamics: Modeling and Simulation of Mechatronic Systems. $3^{\text {rd }}$ Ed. pp. 228-237. John Wiley and Sons, New York, 2000.

[18] F. Grilli, et al. Finite-Element Method Modeling of Superconductors: From 2-D to 3-D. IEEE Transactions on Applied Superconductivity, Vol. 15, NO. 1 March 2005.

[19] J. Rhyner. Magnetic properties and AC losses of superconductors with power law current-voltage characteristics. Physica C, vol. 212, pp. 292300, 1993.

[20] Smythe, William R., Static and Dynamic Electricity, Hemisphere Publishing, New York, 1989, pp. 234, 290-291

[21] H. Woodson and J. Melcher. Electromechanical Dynamics (Part 1).

[22] http://www.ndted.org/GeneralResources/MaterialProperties/ET/Conductivity_Copper.pd $\mathrm{f}$

Mr. Clay S. Hearn obtained the BS and MS degrees in mechanical engineering from the University of Texas at Austin in 2001 and 2008, respectively. He is currently pursuing a Ph.D. in mechanical engineering from the University of Texas with a focus on dynamic system modeling and controls.

Mr. Hearn has been a Research Engineer at the Center for Electromechanics at the University of Texas at Austin since 2002. His work has focused on modeling, analysis, and control of energy storage for various applications, including hybrid vehicles, grid utilities, renewable energies, and pulsed power. He has employed dynamic modeling and control techniques to access potential performance improvements for heavy hybrid vehicles, marine, and microgrid applications by evaluating the addition of energy storage options, including flywheels, lithium ion batteries, and ultracapacitors. Mr. Hearn has also worked extensively on design and evaluation of hydrogen fueled vehicles. Additional work has included FEA structural and thermal modeling and analysis for high-speed composite flywheel energy storage systems and pulse power alternators.

Dr. Siddharth Pratap (M'88-SM'10) received the BS in electrical engineering from the University of Bombay, Bombay, India in 1979, the MS in electrical engineering from the University of Texas at Austin in 1982, and the $\mathrm{PhD}$ in electrical engineering from the University of Texas at Austin in 1996 in the area of power systems and electromagnetics. His research specialty is in the area of electromechanics, computational electromagnetics, and transients in pulsed electrical equipment. He has expertise in the design, analysis, simulation, and experimental performance assessment of electromechanical devices such as linear electrical motors, high average power electrical machines, and pulsed power equipment.

Dr. Pratap served on the integrated product team in the design of the linear induction motor for the Navy's EMALS (Electromagnetic Aircraft Launch System) which is currently being tested and mounted on the next generation of aircraft carriers. He has also worked on the design and analysis of magnetic levitation transportation systems. Recently he has been involved in developing compact, high power systems for mobile Navy and Air Force applications. He also develops 2D and 3D finite element analysis codes to perform transient and steady state, electromagnetic, electrostatic, and thermal analysis on electromechanical equipment including superconducting materials.

Dr. Dongmei Chen received the BS degree from Tsinghua University, Beijing, China, and the MS and PhD degrees, both in mechanical engineering, from the University of Michigan, Ann Arbor, in 2001 and 2006, respectively.

She is currently an Assistant Professor in the Department of Mechanical Engineering at the University of Texas at Austin. Her research interests are in dynamic systems and controls, especially in non-minimum phase, multivariable, and mode switching systems, with applications in wind and solar energy integration, energy storage, and electrical vehicles.

Dr. Chen received a CAREER award from the National Science Foundation (NSF) in 2011. She was a recipient of the University of Michigan Rackham Graduate School Fellowship from 2000 to 2005, and also received several awards for technical excellence while working in the automotive industry.

Dr. Raul G Longoria, P.E., received the BSME and $\mathrm{PhD}$ in mechanical engineering from the University of Texas at Austin in 1985 and 1989, respectively. He joined the Mechanical Engineering Department at the University of Texas at Austin (UT) in 1991 and holds the rank of Professor. His teaching and research interests focus on development and application of multi-disciplinary dynamic system modeling, vehicle system dynamics and controls, electromechanical system modeling and simulation, and medical device design and development. Dr. Longoria is a registered Professional Engineer in the State of Texas, and a member of the ASME, IEEE, SAE, ISTVS, and ASIAO. 